\title{
Bayesian Reordering Model with Feature Selection
}

\section{Abdullah Alrajeh and Mahesan Niranjan}

The Ninth Workshop on Statistical Machine Translation Baltimore, Maryland USA June 26-27, 2014

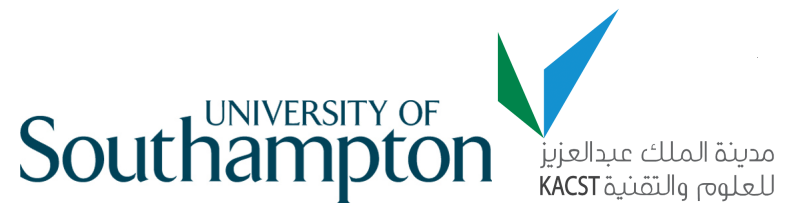




\section{Translation System Overview}

Given a foreign sentence $\mathbf{f}$, the best translation $\mathbf{e}$ is (Brown et al., 1993):

$$
\begin{aligned}
\mathbf{e}_{\text {best }} & =\arg \max _{\mathbf{e}} p(\mathbf{e} \mid \mathbf{f}) \\
& =\arg \max _{\mathbf{e}} \frac{p(\mathbf{f} \mid \mathbf{e}) p(\mathbf{e})}{p(\mathbf{f})} \\
& =\arg \max _{\mathbf{e}} \text { Translation Model } \times \text { Language Model }
\end{aligned}
$$




\section{Translation System Overview}

Given a foreign sentence $\mathbf{f}$, the best translation $\mathbf{e}$ is (Brown et al., 1993):

$$
\begin{aligned}
\mathbf{e}_{\text {best }} & =\arg \max _{\mathbf{e}} p(\mathbf{e} \mid \mathbf{f}) \\
& =\arg \max _{\mathbf{e}} \frac{p(\mathbf{f} \mid \mathbf{e}) p(\mathbf{e})}{p(\mathbf{f})} \\
& =\arg \max _{\mathbf{e}} \text { Translation Model } \times \text { Language Model } \\
\mathbf{e}_{\text {best }} & =\arg \max _{\mathbf{e}}\left\{p_{t}(\mathbf{f} \mid \mathbf{e})^{\lambda_{t}} p_{\text {lm }}(\mathbf{e})^{\lambda_{\text {lm }}} p_{\text {lex }}(\mathbf{f} \mid \mathbf{e})^{\lambda_{\text {lex }}} p_{\text {reo }}(\mathbf{f}, \mathbf{e})^{\lambda_{\text {reo }}} w^{|\mathbf{e}| \lambda_{w}}\right\} \\
& =\arg \max _{\mathbf{e}} \sum_{i} \lambda_{i} \log p_{i}(\mathbf{f}, \mathbf{e})
\end{aligned}
$$




\section{Translation System Overview}

Given a foreign sentence $\mathbf{f}$, the best translation $\mathbf{e}$ is (Brown et al., 1993):

$$
\begin{aligned}
\mathbf{e}_{\text {best }} & =\arg \max _{\mathbf{e}} p(\mathbf{e} \mid \mathbf{f}) \\
& =\arg \max _{\mathbf{e}} \frac{p(\mathbf{f} \mid \mathbf{e}) p(\mathbf{e})}{p(\mathbf{f})} \\
& =\arg \max _{\mathbf{e}} \text { Translation Model } \times \text { Language Model } \\
\mathbf{e}_{\text {best }} & =\arg \max _{\mathbf{e}}\left\{p_{t}(\mathbf{f} \mid \mathbf{e})^{\lambda_{t}} p_{l m}(\mathbf{e})^{\lambda_{\text {lm }}} p_{\text {lex }}(\mathbf{f} \mid \mathbf{e})^{\lambda_{\text {lex }}} p_{\text {reo }}(\mathbf{f}, \mathbf{e})^{\lambda_{\text {reo }}} w^{|\mathbf{e}| \lambda_{w}}\right\} \\
& =\arg \max _{\mathbf{e}} \sum_{i} \lambda_{i} \log p_{i}(\mathbf{f}, \mathbf{e})
\end{aligned}
$$

In general, reordering model is defined as:

$$
p_{\text {reo }}(\mathbf{f}, \mathbf{e})=\prod_{n} p\left(o_{n} \mid \bar{f}_{n}, \bar{e}_{n}\right)=\prod_{n} \frac{h\left(\bar{f}_{n}, \bar{e}_{n}, o_{n}\right)}{\sum_{k} h\left(\bar{f}_{n}, \bar{e}_{n}, o_{k}\right)}
$$




\section{Reordering Models}

$$
\begin{aligned}
& \begin{array}{ll|l|l|l}
\text { Foreign sentence } \mathbf{f}: & \bar{f}_{1} & \bar{f}_{2} & \bar{f}_{3}
\end{array} . \\
& \begin{array}{ll|l|l|}
\text { English sentence } \mathbf{e}: & \bar{e}_{1} & \bar{e}_{3} & \bar{e}_{2}
\end{array} . \\
& p_{\text {reo }}(\mathbf{f}, \mathbf{e})=p\left(o_{1}=\operatorname{mono} \mid \bar{f}_{1}, \bar{e}_{1}\right) \times p\left(o_{2}=\operatorname{swap} \mid \bar{f}_{2}, \bar{e}_{2}\right) \times p\left(o_{3}=\text { other } \mid \bar{f}_{3}, \bar{e}_{3}\right)
\end{aligned}
$$




\section{Reordering Models}

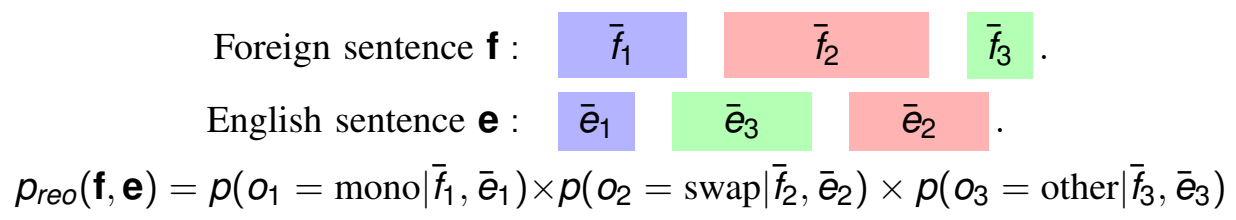

- Lexicalized Reordering Model (Tillmann, 2004; Kumar and Byrne, 2005; Koehn et al., 2005; Galley and Manning, 2008)

$$
p\left(o_{k} \mid \bar{f}_{n}, \bar{e}_{n}\right)=\frac{\operatorname{count}\left(\bar{f}_{n}, \bar{e}_{n}, o_{k}\right)}{\sum_{k^{\prime}} \operatorname{count}\left(\bar{f}_{n}, \bar{e}_{n}, o_{k^{\prime}}\right)}
$$




\section{Reordering Models}

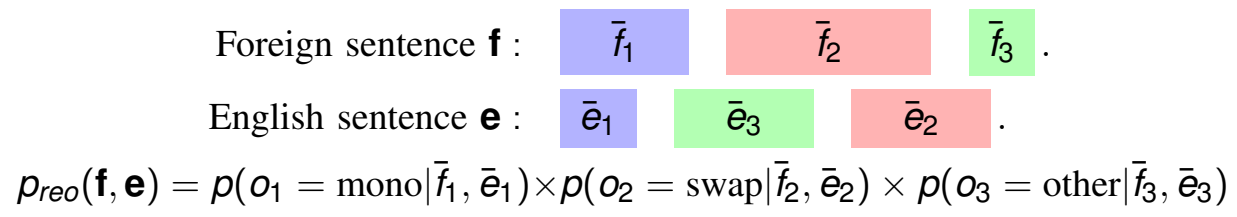

- Lexicalized Reordering Model (Tillmann, 2004; Kumar and Byrne, 2005; Koehn et al., 2005; Galley and Manning, 2008)

$$
p\left(o_{k} \mid \bar{f}_{n}, \bar{e}_{n}\right)=\frac{\operatorname{count}\left(\bar{f}_{n}, \bar{e}_{n}, o_{k}\right)}{\sum_{k^{\prime}} \operatorname{count}\left(\bar{f}_{n}, \bar{e}_{n}, o_{k^{\prime}}\right)}
$$

- Discriminative Reordering Model (Zens and Ney, 2006; Xiong et al., 2006; Nguyen et al., 2009; Xiang et al., 2011; Ni et al., 2011)

$$
p\left(o_{k} \mid \bar{f}_{n}, \bar{e}_{n}\right)=\frac{\exp \left(\mathbf{w}_{k}^{T} \phi\left(\bar{f}_{n}, \bar{e}_{n}\right)\right)}{\sum_{k^{\prime}} \exp \left(\mathbf{w}_{k^{\prime}}^{T} \phi(\bar{f}, \bar{e})\right)} \equiv \frac{\exp \left(\mathbf{w}^{T} \phi\left(\bar{f}_{n}, \bar{e}_{n}, o_{k}\right)\right)}{\sum_{k^{\prime}} \exp \left(\mathbf{w}^{T} \phi\left(\bar{f}, \bar{e}, o_{k^{\prime}}\right)\right)}
$$




\section{Feature Extraction}

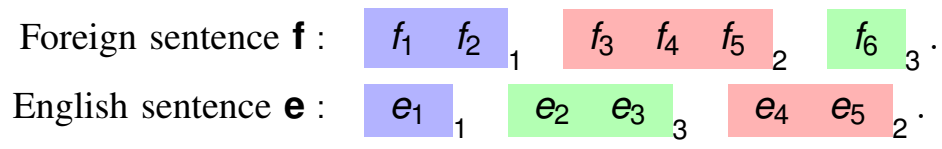




\section{Feature Extraction}

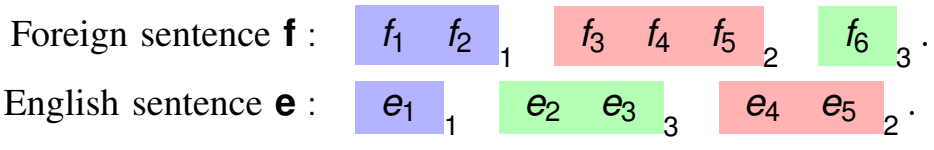

Extracted phrase pairs :

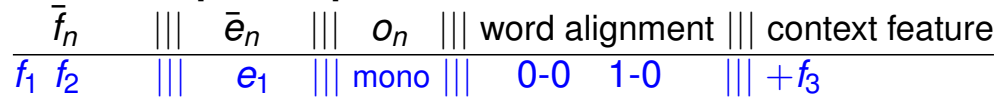

$f_{3} f_{4} f_{5} \quad\left\|e_{4} e_{5} \quad\right\| \mid$ swap $\| \mid \begin{array}{llllll}0-1 & 2-0 & \| & -f_{2} & +f_{6}\end{array}$

$f_{6} \quad \| \quad|| e_{2} e_{3} \quad|| \mid$ other || $\mid \begin{array}{llll}0-0 & 0-1 & \| & -f_{5}\end{array}$ 


\section{Feature Extraction}

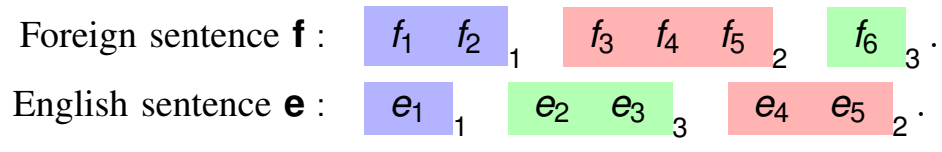

Extracted phrase pairs :

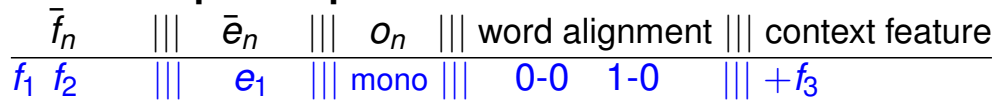

$f_{3} f_{4} f_{5} \quad\left\|\left|e_{4} e_{5} \quad\left\||\operatorname{swap} \|| \mid \begin{array}{llllll}0-1 & 2-0 & \|-f_{2} & +f_{6}\end{array}\right.\right.\right.$

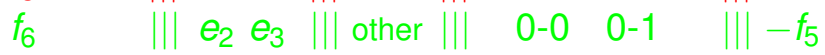

All linguistic features:

$\left(f_{1} \& e_{1}\right)^{1}\left(f_{2} \& e_{1}\right)^{2}\left(+f_{3}\right)^{3}\left(f_{3} \& e_{5}\right)^{4}\left(f_{5} \& e_{4}\right)^{5}\left(-f_{2}\right)^{6}\left(+f_{6}\right)^{7}\left(f_{6} \& e_{2}\right)^{8}\left(f_{6} \& e_{3}\right)^{9}\left(-f_{5}\right)^{10}$

Bag-of-words representation ( $0=$ not exist):

$\frac{\phi\left(\bar{f}_{n}, \bar{e}_{n}\right) \quad 12345678910}{\phi\left(\bar{f}_{1}, \bar{e}_{n}\right)=1110000000}$

$\phi\left(\bar{f}_{1}, \bar{e}_{1}\right)=1110000000$

$\phi\left(\bar{f}_{2}, \bar{e}_{2}\right)=0001111000$

$\phi\left(\bar{f}_{3}, \bar{e}_{3}\right)=000000000111$ 


\section{The Proposed Reordeing Model}

\section{Naive Bayes}

$$
p\left(o_{k} \mid \bar{f}_{n}, \bar{e}_{n}\right)=\frac{p\left(\bar{f}_{n}, \bar{e}_{n} \mid o_{k}\right) p\left(o_{k}\right)}{\sum_{k^{\prime}} p\left(\bar{f}_{n}, \bar{e}_{n} \mid o\right) p\left(o_{k^{\prime}}\right)} .
$$

Multinomial distribution:

$$
p\left(\bar{f}_{n}, \bar{e}_{n} \mid \mathbf{q}_{k}\right)=C \prod_{m}^{M} q_{k m}{ }^{\phi_{m}\left(\bar{f}_{n}, \bar{e}_{n}\right)}
$$




\section{The Proposed Reordeing Model}

\section{Naive Bayes}

$$
p\left(o_{k} \mid \bar{f}_{n}, \bar{e}_{n}\right)=\frac{p\left(\bar{f}_{n}, \bar{e}_{n} \mid o_{k}\right) p\left(o_{k}\right)}{\sum_{k^{\prime}} p\left(\bar{f}_{n}, \bar{e}_{n} \mid o\right) p\left(o_{k^{\prime}}\right)} .
$$

Multinomial distribution:

$$
p\left(\bar{f}_{n}, \bar{e}_{n} \mid \mathbf{q}_{k}\right)=C \prod_{m}^{M} q_{k m} \phi_{m}\left(\bar{f}_{n}, \bar{e}_{n}\right)
$$

Maximum-likelihood estimation:

$$
q_{k m}^{*}=\arg \max _{\mathbf{q}_{k}} \prod_{n}^{N_{k}} p\left(\bar{f}_{n}, \bar{e}_{n} \mid \mathbf{q}_{k}\right)=\frac{\sum_{n}^{N_{k}} \phi_{m}\left(\bar{f}_{n}, \bar{e}_{n}\right)}{\sum_{m^{\prime}}^{M} \sum_{n}^{N_{k}} \phi_{m^{\prime}}\left(\bar{f}_{n}, \bar{e}_{n}\right)} .
$$




\section{The Proposed Reordeing Model}

\section{Naive Bayes}

$$
p\left(o_{k} \mid \bar{f}_{n}, \bar{e}_{n}\right)=\frac{p\left(\bar{f}_{n}, \bar{e}_{n} \mid o_{k}\right) p\left(o_{k}\right)}{\sum_{k^{\prime}} p\left(\bar{f}_{n}, \bar{e}_{n} \mid o\right) p\left(o_{k^{\prime}}\right)} .
$$

Multinomial distribution:

$$
p\left(\bar{f}_{n}, \bar{e}_{n} \mid \mathbf{q}_{k}\right)=C \prod_{m}^{M} q_{k m} \phi_{m}\left(\bar{f}_{n}, \bar{e}_{n}\right)
$$

Maximum-likelihood estimation:

$$
q_{k m}^{*}=\arg \max _{\mathbf{q}_{k}} \prod_{n}^{N_{k}} p\left(\bar{f}_{n}, \bar{e}_{n} \mid \mathbf{q}_{k}\right)=\frac{\sum_{n}^{N_{k}} \phi_{m}\left(\bar{f}_{n}, \bar{e}_{n}\right)}{\sum_{m^{\prime}}^{M} \sum_{n}^{N_{k}} \phi_{m^{\prime}}\left(\bar{f}_{n}, \bar{e}_{n}\right)} .
$$

Maximum a posteriori (MAP) estimation:

$q_{k m}^{*}=\arg \max _{\mathbf{q}_{k}} \prod_{n}^{N_{k}} p\left(\bar{f}_{n}, \bar{e}_{n} \mid \mathbf{q}_{k}\right) p\left(\mathbf{q}_{k} \mid \boldsymbol{\alpha}\right)=\frac{\alpha-1+\sum_{n}^{N_{k}} \phi_{m}\left(\bar{f}_{n}, \bar{e}_{n}\right)}{M(\alpha-1)+\sum_{m^{\prime}}^{M} \sum_{n}^{N_{k}} \phi_{m^{\prime}}\left(\bar{f}_{n}, \bar{e}_{n}\right)}$ 


\section{The Proposed Reordeing Model}

Bayesian Naive Bayes (Barber, 2012)

$$
p\left(o_{k} \mid \bar{f}_{n}, \bar{e}_{n}\right)=\frac{p\left(\bar{f}_{n}, \bar{e}_{n} \mid o_{k}\right) p\left(o_{k}\right)}{\sum_{k^{\prime}} p\left(\bar{f}_{n}, \bar{e}_{n} \mid o\right) p\left(o_{k^{\prime}}\right)} .
$$

\section{Full Bayesian Inference}

Multinomial-Dirichlet

$$
\begin{aligned}
& p\left(\bar{f}_{n}, \bar{e}_{n} \mid o_{k}\right)=\int p\left(\bar{f}_{n}, \bar{e}_{n} \mid \mathbf{q}_{k}\right) p\left(\mathbf{q}_{k} \mid \alpha_{k}\right) \mathrm{d} \mathbf{q}_{k} \\
& =C \frac{\Gamma\left(\sum_{m} \alpha_{k m}\right)}{\prod_{m} \Gamma\left(\alpha_{k m}\right)} \frac{\prod_{m} \Gamma\left(\alpha_{k m}+\phi_{m}\left(\bar{f}_{n}, \bar{e}_{n}\right)\right)}{\Gamma\left(\sum_{m} \alpha_{k m}+\phi_{m}\left(\bar{f}_{n}, \bar{e}_{n}\right)\right)} \\
& p\left(\mathbf{q}_{k} \mid \alpha_{k}\right)=\frac{p\left(\mathbf{q}_{k} \mid \alpha\right) \prod_{n}^{N_{k}} p\left(\bar{f}_{n}, \bar{e}_{n} \mid \mathbf{q}_{k}\right)}{\int p\left(\mathbf{q}_{k} \mid \alpha\right) \prod_{n}^{N_{k}} p\left(\bar{f}_{n}, \bar{e}_{n} \mid \mathbf{q}_{k}\right) \mathrm{d} \mathbf{q}_{k}}
\end{aligned}
$$

Prior and Posterior

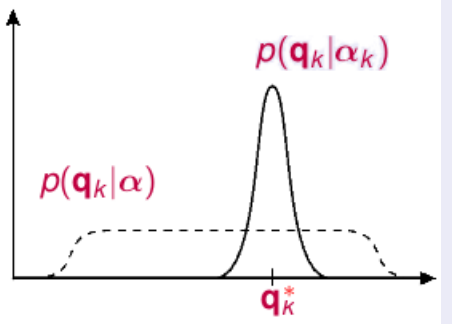

$$
\boldsymbol{\alpha}_{k}=\boldsymbol{\alpha}+\sum_{n}^{N_{k}} \phi\left(\bar{f}_{n}, \bar{e}_{n}\right)
$$




\section{Classification Results}

3-class problem: mono, swap, other

Table: Arabic-English MultiUN corpus (Eisele and Chen, 2010)

\begin{tabular}{c|c|c}
\hline Statistics & Arabic & English \\
\hline Sentence Pairs & \multicolumn{2}{|c}{$9.7 \mathrm{M}$} \\
\hline Running Words & $255.5 \mathrm{M}$ & $285.7 \mathrm{M}$ \\
Word/Line & 22 & 25 \\
Vocabulary Size & $677 \mathrm{~K}$ & $410 \mathrm{~K}$ \\
\hline
\end{tabular}




\section{Classification Results}

\section{3-class problem: mono, swap, other}

Table: Arabic-English MultiUN corpus (Eisele and Chen, 2010)

\begin{tabular}{c|c|c}
\hline Statistics & Arabic & English \\
\hline Sentence Pairs & \multicolumn{2}{|c}{$9.7 \mathrm{M}$} \\
\hline Running Words & $255.5 \mathrm{M}$ & $285.7 \mathrm{M}$ \\
Word/Line & 22 & 25 \\
Vocabulary Size & $677 \mathrm{~K}$ & $410 \mathrm{~K}$ \\
\hline
\end{tabular}

Table: Error rate based on 3-fold cross-validation

\begin{tabular}{l|c}
\hline Classifier & Error Rate \\
\hline Lexicalized model & $25.2 \%$ \\
Bayes-MAP estimate & $\mathbf{1 9 . 5 3 \%}$ \\
Bayes-Bayesian inference & $20.13 \%$ \\
\hline
\end{tabular}




\section{Feature Selection}

\section{Normalized mutual information (Estevez et al., 2009):}

$$
I_{\text {norm }}(X ; Y)=\frac{I(X ; Y)}{\min (H(X), H(Y))} \text {. }
$$
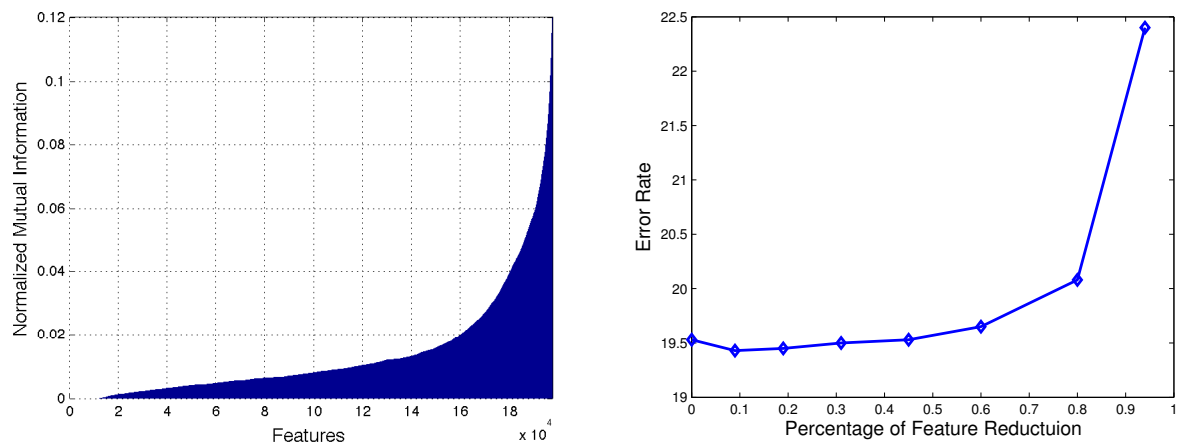


\section{Translation Results}

Table: NIST test sets (4 references for each Arabic sentence)

\begin{tabular}{cc|c|c}
\hline \multicolumn{2}{c|}{ Evaluation Set } & Arabic & English \\
\hline NIST MT06 & sentences & 1797 & 7188 \\
& words & $49 \mathrm{~K}$ & $223 \mathrm{~K}$ \\
\hline NIST MT08 & sentences & 813 & 3252 \\
& words & $25 \mathrm{~K}$ & $117 \mathrm{~K}$ \\
\hline
\end{tabular}




\section{Translation Results}

Table: NIST test sets (4 references for each Arabic sentence)

\begin{tabular}{cc|c|c}
\hline \multicolumn{2}{c|}{ Evaluation Set } & Arabic & English \\
\hline NIST MT06 & sentences & 1797 & 7188 \\
& words & $49 \mathrm{~K}$ & $223 \mathrm{~K}$ \\
\hline NIST MT08 & sentences & 813 & 3252 \\
& words & $25 \mathrm{~K}$ & $117 \mathrm{~K}$ \\
\hline
\end{tabular}

Table: BLEU Score (Papineni et al., 2002)

\begin{tabular}{l|c|c|c|c}
\hline Translation System & ReoM Size & Speed & MT06 & MT08 \\
\hline Baseline & - & - & 28.92 & 32.13 \\
BL + Lexicalized ReoM & $604 \mathrm{MB}$ & $2.2 \mathrm{sec} / \mathrm{s}$ & 30.86 & 34.22 \\
BL + Bayes-MAP ReoM & $18 \mathrm{MB}$ & $2.6 \mathrm{sec} / \mathrm{s}$ & $\mathbf{3 1 . 2 1}$ & $\mathbf{3 4 . 7 2}$ \\
BL + Bayes-Baysien ReoM & $18 \mathrm{MB}$ & $2.6 \mathrm{sec} / \mathrm{s}$ & $\mathbf{3 1 . 2 0}$ & $\mathbf{3 4 . 6 9}$ \\
\hline
\end{tabular}




\section{Thank you for your attention.}

